\title{
Factors in seminal plasma of bulls that affect the viability and motility of spermatozoa
}

\author{
Joan W. Baas, P. C. Molan and P. Shannon* \\ School of Science, University of Waikato, Hamilton and \\ ${ }^{*}$ New Zealand Dairy Board Artificial Breeding Centre, Newstead, R.D. 4, Hamilton, New Zealand
}

\begin{abstract}
Summary. When ejaculated bovine semen was washed twice with Ficoll the spermatozoa, resuspended in buffer, became immotile. Motility could be restored by addition of seminal plasma from vasectomized bulls and by addition of both bovine serum albumin (BSA) and theophylline. Motility could be restored and maintained at $37^{\circ} \mathrm{C}$ to a variable extent with BSA alone. When this motility ceased it could be revived with theophylline or seminal plasma. When spermatozoa, inactivated by washing with Ficoll, were reactivated with seminal plasma the time that motility lasted at $37^{\circ} \mathrm{C}$ before it was irreversibly lost depended on the concentration of seminal plasma: the more seminal plasma added, the shorter the duration of motility. This suggested that seminal plasma contained separate factors which restored motility and led to permanent inactivation of the spermatozoa. A motility-stimulating factor was present in the low molecular weight fraction of seminal plasma which had been passed through an ultrafilter of retention $M_{r} 500$ and the damaging effect was confined to the high molecular weight non-dialysable fraction.
\end{abstract}

\section{Introduction}

It has long been known that seminal plasma functions to transport and sustain motility of spermatozoa (Mann, 1964). However, seminal plasma, particularly that of bulls, is also detrimental to sperm survival (Shannon, 1965a; Dott, 1974). Various techniques have been used to study these effects. Eliasson, Johnsen \& Lindholmer (1974), who collected fluid from the human prostate and seminal vesicles by the 'split-ejaculate technique', concluded that prostatic fluid has a beneficial effect while vesicular fluid has an adverse effect on motility and survival of the spermatozoa. Dott, Harrison \& Foster (1979) examined the effects of epididymal plasma, seminal plasma and serum albumin on washed epididymal spermatozoa and concluded that seminal plasma is detrimental to survival. A 'sperm motility factor' has been obtained from the spermatozoa of several species (Bavister \& Yanagimachi, 1977), epididymal fluid (Bavister, Rogers \& Yanagimachi, 1978), human serum (Bavister, 1975) and hamster and bovine adrenal glands (Bavister, Chen \& Fu, 1979). This factor stimulates and sustains motility and fertilizing ability of hamster epididymal spermatozoa in vitro, but catecholamines as well as 'sperm motility factor' are required for optimal motility stimulation (Bavister et al., 1979).

Spermatozoa from ejaculated bull semen, inactivated by Ficoll washing (Harrison, 1976), have their motility restored by seminal plasma (Dott et al., 1979) but bovine serum albumin has produced variable results. Dott et al. (1979) ascribe a sperm motility-stimulating role to serum albumin but Harrison, Dott \& Foster (1978) found that although bovine serum albumin was beneficial to motility of boar, rabbit, ram and stallion semen it had little effect on motility of bull spermatozoa washed in Ficoll. Since phosphodiesterase inhibitors have been shown to stimulate motility of spermatozoa (Garbers \& Kopf, 1980), we have examined the effect of theophylline in combination with BSA on the motility of bull spermatozoa. 


\section{Materials and Methods}

Reagents. Bovine serum albumin, theophylline and Ficoll were obtained from Sigma Chemical Co. (St. Louis, Missouri, U.S.A.).

Diluent. In all studies spermatozoa were suspended in a diluent composed of citrate buffer with glucose, glycerol, glycine, caproic acid, catalase and antibiotics (Shannon, 1965b).

Statistical techniques. Data were analysed by the techniques for analysis of variance outlined by Snedecor \& Cochran (1972). When a number of treatments have been compared we have shown the standard error of the treatment (s.e.t.), which is defined as $\sqrt{\mathrm{EMS}} / n$ where EMS is error mean square and $n$ the number of replicates/treatment. When, as for example in Trials 1 and 2 , inactivated semen was initially reactivated with BSA before incubation and treatment with different levels of a compound, the standard error of the mean (s.e.m.) of the BSA treatment has also been shown.

Washing of spermatozoa. Ejaculated bull semen collected by means of an artificial vagina was immediately added to $10 \mathrm{ml}$ semen diluent and $2 \mathrm{ml}$ aliquants were then carefully layered on top of $10 \mathrm{ml} \mathrm{Ficoll}(7 \%$ in diluent) in centrifuge tubes. The tubes were centrifuged at $475 \mathrm{~g}$ for $15 \mathrm{~min}$ and the supernatant layers were removed by aspiration. The wash was then repeated and the washed spermatozoa diluted to a concentration of $200 \times 10^{6} / \mathrm{ml}$ and stored at room temperature overnight to allow a full day for testing the spermatozoa. Sperm concentrations were estimated using an absorbtiometer which had been previously calibrated with haemocytometer counts (Digital Ultraviolet Spectrophotometer, Cecil Instruments Ltd, Cambridge, U.K.).

Evaluation of motility of spermatozoa. Spermatozoa, at a concentration of $10 \times 10^{6} / \mathrm{ml}$, were suspended in a mixture of diluent and the components to be tested to give a total volume of $5 \mathrm{ml}$. The spermatozoa were incubated at $37^{\circ} \mathrm{C}$ in stoppered glass tubes. Motility measurements were made by taking a sample, examining it visually at $37^{\circ} \mathrm{C}$ using a microscope, and estimating the percentage that showed rapid progressive motility. Estimations of motility were made every hour and motility was said to have ceased when no more than $5 \%$ of the spermatozoa showed any movement. When a compound, for example BSA or theophylline, caused activation of spermatozoa, the percentage of spermatozoa motile was assessed 5-10 min after addition of the compound.

Preparation of fractions. Seminal plasma was obtained by pooling ejaculates collected with an artificial vagina from vasectomized bulls. A sample was retained for testing and $50 \mathrm{ml}$ were dialysed against an equal volume of distilled water for $24 \mathrm{~h}$ using dialysis tubing (Visking Company, Chicago, Illinois, U.S.A.) with a molecular weight cut-off of 10000 . The diffusate was retained and the process was repeated with a change of distilled water a further three times. The dialysed seminal plasma (the retentate) was retained for testing after dialysis against 3 times its volume of diluent solution for $4 \mathrm{~h}$ to achieve an osmotic pressure equivalent to that of seminal plasma.

The pooled diffusate was ultrafiltered through a filter of nominal retention of $M_{r} 500$ (Amicon Corp., Lexington, Massachusetts, U.S.A.). The material retained, $\sim 10 \mathrm{ml}$, was made up to the original volume of the seminal plasma with diluent. The ultrafiltrate was concentrated to the original volume of the seminal plasma by rotary evaporation.

\section{Results}

Evaluation of the assays

Assessment of motility-stimulating activity. Seminal plasma was added to inactivated spermatozoa suspended in diluent to give a final concentration of $0,2,5,10$ or $20 \%$. Stimulation of motility occurred in all tests and the percentage of motile spermatozoa was proportional to the concentration of seminal plasma added: $0,35,52 \cdot 5,67 \cdot 5$ and $75 \%$ respectively, When BSA was added, to a final concentration of $0 \cdot 1,0 \cdot 2,0 \cdot 5,1$ or $2 \%$, to inactivated spermatozoa suspended in 
diluent motility was always restored, to $46,46,57 \cdot 5,63 \cdot 8$ and $63.8 \%$ respectively, with maximum activity being reached with a concentration of $1 \% \mathrm{BSA}$.

In two trials the percentage of motile spermatozoa reactivated by suspending spermatozoa in diluent plus $1 \%$ BSA was $41.7 \pm 33.0(n=3)$ and $50 \pm 23.5(n=4)$. Motility at $37^{\circ} \mathrm{C}$ ceased after $7.6 \mathrm{~h}$ in Trial 1 (range $2-18 \mathrm{~h}$ ) and after $5.1 \mathrm{~h}$ in Trial 2 (range $2-9 \mathrm{~h}$ ). The mean of the two trials combined was $46.4 \%$ motile and survival for $6.1 \mathrm{~h}$. The correlation between percentage spermatozoa reactivated and survival was $0.814(P<0.05)$. When motility ceased, addition of further BSA did not restore motility but spermatozoa were reactivated by theophylline (see Table 1) or seminal plasma (see Table 2) in a concentration-dependent manner.

Table 1. Action of theophylline on inactivated bovine spermatozoa when motility with BSA alone* has ceased

\begin{tabular}{|c|c|}
\hline $\begin{array}{l}\text { Conc. } \\
\text { theophylline }(\%)\end{array}$ & $\begin{array}{l}\% \text { Motility on } \\
\text { reactivation } \dagger\end{array}$ \\
\hline 0.2 & $68 \cdot 3$ \\
\hline $0 \cdot 1$ & $65 \cdot 0$ \\
\hline 0.05 & 60.0 \\
\hline 0.02 & $53 \cdot 3$ \\
\hline 0.01 & $21 \cdot 7 \ddagger$ \\
\hline \multicolumn{2}{|c|}{$\begin{array}{l}\text { * Motility when BSA was first added was } 41 \cdot 7 \pm \\
0 \text { (s.e.m.) } \% \text {. } \\
\dagger \text { s.e.t. } \pm 6 \cdot 3 \text {. Difference between theophylline } \\
\text { ncentrations } P<0.01 ; n=3 \text {. } \\
\pm \text { Significantly different from all other }\end{array}$} \\
\hline
\end{tabular}

Table 2. Action of seminal plasma on inactivated bovine spermatozoa when motility with BSA alone* has ceased

\begin{tabular}{cc}
\hline $\begin{array}{c}\text { Conc. } \\
\text { seminal plasma }(\%)\end{array}$ & $\begin{array}{c}\% \text { Motility on } \\
\text { reactivation } \dagger\end{array}$ \\
\hline 20 & $67 \cdot 5^{\mathrm{a}}$ \\
10 & $56 \cdot 3^{\mathrm{a}}$ \\
5 & $40 \cdot 0^{\mathrm{b}}$ \\
2 & $17 \cdot 5^{\mathrm{c}}$ \\
\hline
\end{tabular}

\footnotetext{
* Motility when BSA was first added was $50 \pm 13.6$ (s.e.m.) $\%$

$\dagger$ s.e.t. $\pm 4 \cdot 8$. Difference between seminal plasma concentrations $P<0.01 ; n=4$. Values with different superscript letters are significantly different.
}

When theophylline was added in a concentration of $0.2 \%$ to spermatozoa inactivated by washing, no reactivation occurred, indicating that washing in Ficoll must remove from the spermatozoa the components which, like BSA, maintain motility in ejaculated spermatozoa. (It was found that two Ficoll washes were needed to inactivate the spermatozoa: after one wash spermatozoa suspended in diluent retained some motility.)

To test further the ability of BSA alone to restore motility after Ficoll washing, spermatozoa were dialysed overnight at a concentration of $20 \times 10^{6} / \mathrm{ml}$ against 3 volumes of diluent to remove the theophylline-like activation. In all tests subsequent reactivation with BSA after dialysis was considerably reduced compared with a control of non-dialysed spermatozoa from the same batch: in some tests no reactivation at all occurred. 
Assessment of damaging effect. Initially, seminal plasma was added to inactivated spermatozoa, suspended in diluent, to achieve final concentrations of $0,2,5,10$ or $20 \%$. The motility that resulted varied with the concentration of seminal plasma added $(P<0.01)$ (see Table 3 ) and was maintained for various periods of time. When motility ceased the time was recorded and BSA and theophylline were added to give concentrations of $1 \%$ and $0 \cdot 1 \%$ respectively. The results are shown in Table 3. The more seminal plasma present the shorter the duration of motility, but some reactivation by BSA and theophylline was seen when lower concentrations of seminal plasma were used.

Table 3. Effect of seminal plasma on the initial motility and duration of activity of inactivated bovine spermatozoa

\begin{tabular}{|c|c|c|c|}
\hline $\begin{array}{l}\text { Conc. seminal } \\
\text { plasma }(\%)\end{array}$ & $\begin{array}{l}\% \text { Motility after } \\
\text { initial } \\
\text { reactivation* } \\
(n=4)\end{array}$ & $\begin{array}{l}\text { Duration of } \\
\text { motility }(h) \dagger \\
\quad(n=4)\end{array}$ & $\begin{array}{l}\% \text { Motility after } \\
\text { subsequent } \\
\text { reactivation by } \\
\text { BSA and } \\
\text { theophylline } \neq \\
(n=3)\end{array}$ \\
\hline 20 & 75 & 2.9 & 0 \\
\hline 10 & 67.5 & 3.9 & $1 \cdot 7$ \\
\hline 5 & $52 \cdot 5$ & $5 \cdot 5$ & $13 \cdot 7$ \\
\hline 2 & $35 \cdot 0$ & $7 \cdot 3$ & $26 \cdot 7$ \\
\hline 0 & 0 & - & $60 \cdot 0 \S$ \\
\hline
\end{tabular}

* s.e.t. $\pm 2 \cdot 2$. Difference $P<0.01(0$ omitted $)$. All differences significant.

† s.e.t. \pm 0.7 . Difference $P<0.01 ; 20 \%$ differs significantly from 5 and $2 \% ; 10 \%$ differs significantly from $2 \%$.

$\ddagger$ s.e.t. $\pm 9 \cdot 1$. Difference $P<0.01 ; 20 \%$ differs significantly from 5,2 and $0 \% ; 10$ and $5 \%$ differ significantly from 2 and $0 \%$.

$\S$ Reactivated at the same time as samples with $2 \%$ seminal plasma.

To assess the damaging effect independently of a motility-stimulating effect the assay was repeated with the BSA and theophylline added at the start to achieve maximal initial reactivation of motility (75-81.3\%). No restoration of motility was achieved by the further addition of BSA and theophylline (Table 4). The more seminal plasma present, the shorter the duration of motility.

Table 4. Effect of seminal plasma $(n=4)$ on the duration of activity of inactivated bovine spermatozoa reactivated with BSA and theophylline

\begin{tabular}{ccc}
\hline $\begin{array}{c}\text { Conc. seminal } \\
\text { plasma }(\%)\end{array}$ & $\begin{array}{c}\text { \% Motility after } \\
\text { reactivation* }\end{array}$ & $\begin{array}{c}\text { Duration of } \\
\text { motility (h) } \dagger\end{array}$ \\
\hline 20 & $77 \cdot 5$ & $4 \cdot 0$ \\
10 & $81 \cdot 3$ & $5 \cdot 1$ \\
5 & $75 \cdot 0$ & $6 \cdot 4$ \\
2 & $77 \cdot 5$ & $11 \cdot 2$ \\
0 & $77 \cdot 5$ & $15 \cdot 8$ \\
\hline
\end{tabular}

* s.e.t. \pm 2.9 (N.S.).

$\dagger$ s.e.t. $\pm 2 \cdot 2 . P<0 \cdot 025 ; 20 \%$ significantly different from 2 and $0 \% ; 10$ and $5 \%$ significantly different from $0 \%$.

\section{Evaluation of fractions of seminal plasma}

Fractions of seminal plasma were assayed for their motility-stimulating effect and for their damaging effect. The final volume of each fraction was adjusted to be the same as the original seminal plasma volume. All assays were done at a final concentration of $10 \%$ of the fraction tested. 
To locate the motility-stimulating effect, samples of the fractions were added to spermatozoa in which motility previously stimulated with $1 \%$ BSA had ceased. There was $75 \%$ motility with whole seminal plasma, $7.5 \%$ with the retentate of dialysed seminal plasma, $7.5 \%$ with the material retained after ultrafiltration of pooled diffusate and $72.6 \%$ with the material which passed through the ultrafilter (s.e.t. $\pm 2 \cdot 4, n=4$ ). Most of the motility-stimulating components of seminal plasma were contained in the material which passed through the ultrafilter.

To locate the damaging effect, a separate assay was performed in which inactivated spermatozoa were incubated with the seminal plasma fractions at a final concentration of $10 \%$ with BSA $(1 \%)$ and theophylline $(0 \cdot 1 \%)$ added at the start of incubation. Damage was assessed by the duration of motility. The damaging effect was confined to the non-dialysable high molecular weight fraction (Table 5).

Table 5. Effect of fractions of seminal plasma $(n=4)$ on the duration of activity of inactivated spermatozoa reactivated with BSA and theophylline

\begin{tabular}{lcr}
\hline & $\begin{array}{c}\text { \% Motility on } \\
\text { reactivation* }\end{array}$ & $\begin{array}{r}\text { Duration of } \\
\text { motility (h) } \dagger\end{array}$ \\
\hline Whole seminal plasma & $77 \cdot 5$ & $7 \cdot 5$ \\
Retentate of dialysed seminal plasma $\left(M_{r}>10000\right)$ & $74 \cdot 0$ & $5 \cdot 5$ \\
Ultrafilter retentate $\left(500<M_{r}<10000\right)$ & $78 \cdot 8$ & $21 \cdot 8$ \\
Ultrafiltrate $\left(M_{r}<500\right)$ & $78 \cdot 8$ & $21 \cdot 8$ \\
Control (diluent) & $78 \cdot 8$ & $20 \cdot 3$ \\
\hline
\end{tabular}

* s.e.t. $\pm 2 \cdot 5$ (N.S.).

$\dagger$ s.e.t. $\pm 2 \cdot 0 . P<0 \cdot 01$; whole seminal plasma and dialysis retentate significantly different from all other treatments.

To examine further the motility-stimulating effect, the ultrafiltered material at various concentrations was added to samples of washed spermatozoa in which motility stimulated with $1 \%$ BSA had ceased. Almost full activation was achieved with 5,10 and $20 \%$ of the fraction $(67,72$ and $76 \%$ respectively) as compared to the gradual increase in activation with increase in seminal plasma concentration, suggesting that seminal plasma contains a factor which interferes with the motilitystimulating effect. In addition sustained motility lasting several hours was observed with the low molecular weight fraction whereas with whole seminal plasma motility declined rapidly, particularly with levels above $10 \%$.

\section{Discussion}

The double washing of ejaculated bovine spermatozoa in Ficoll is a useful technique for their study; the spermatozoa can be obtained free of seminal plasma in a condition in which they are immotile but revivable for a period of time. This provides an excellent test for the factors affecting motility and viability of bull spermatozoa. The assays clearly demonstrated the damaging effect of seminal plasma: it causes spermatozoa to lose their activity sooner, the inactivation being permanent. This damaging effect is confined to the high molecular weight fraction of seminal plasma. The occurrence of separate factors that sustain and maintain the motility of washed spermatozoa was demonstrated also: bovine spermatozoa require a high molecular weight factor which can be replaced by bovine serum albumin and a low molecular weight factor with a theophylline-like effect. After washing in Ficoll the spermatozoa still possessed some low molecular weight motilitystimulating component. The variable degree of stimulation of motility obtained with BSA in these experiments, the reduction in stimulation after dialysis of spermatozoa and the poor stimulation of motility of bull spermatozoa with BSA reported by Harrison et al. (1978) could be explained by a variation in the amount of low molecular weight motility-stimulating component retained by 
bovine spermatozoa after washing in Ficoll. Although dialysis reduces the activation by BSA, sometimes completely, it is not convenient for routine assays.

The low molecular weight component is probably present in or bound to spermatozoa and is gradually lost during dilution and incubation with BSA until motility ceases. At this point motility can be restored by the addition of theophylline or the low molecular weight fraction of seminal plasma. This low molecular weight factor has much in common with the 'sperm motility factor' extracted from washed epididymal spermatozoa by Bavister \& Yanagimachi (1977). Further study of this and the other factors affecting the motility and viability of bull spermatozoa will be aided by the establishment of the techniques described in this paper. These techniques enable the two types of motility-stimulating effect and the damaging effect to be assayed separately without interference of one effect with another.

We thank B. Curson for assistance with this work, and the Medical Research Council of New Zealand for support in the form of a Research Scholarship for J.W.B.

\section{References}

Bavister, B.D. (1975) Properties of the sperm motilitystimulating component derived from human serum. J. Reprod. Fert. 43, 363-366.

Bavister, B.D. \& Yanagimachi, R. (1977) The effects of sperm extracts and energy sources on the motility and acrosome reaction of hamster spermatozoa in vitro. Biol. Reprod. 16, 228-237.

Bavister, B.D., Rogers, B.J. \& Yanagimachi, R. (1978) The effects of cauda epididymal plasma on the motility and acrosome reaction of hamster and guinea pig spermatozoa in vitro. Biol. Reprod. 19, 358363.

Bavister, B.D., Chen, A.F. \& Fu, P.C. (1979) Catecholamine requirement for hamster sperm motility in vitro. J. Reprod. Fert. 56, 507-513.

Dott, H.M. (1974) The effects of bovine seminal plasma on the impedance change frequency and glycolysis of bovine epididymal spermatozoa. J. Reprod. Fert. 38, $147-156$.

Dott, H.M., Harrison, R.A.P. \& Foster, G.C.A. (1979) The maintenance of motility and the surface properties of epididymal spermatozoa from bull, rabbit and ram in homologous seminal and epididymal plasma. J. Reprod. Fert. 55, 113-124.

Eliasson, R., Johnsen, O. \& Lindholmer, C. (1974) Effects of seminal plasma on some functional properties of human spermatozoa. In Male Fertility, Sterility, ch. 5, pp. 107-122. Eds R. E. Mancini \& L. Martini. Academic Press, New York.

Garbers, D.L. \& Kopf, G.S. (1980) The regulation of spermatozoa by calcium and cyclic nucleotides. $A d v$. cyclic Nucleotide Res. 13, 251-306.

Harrison, R.A.P. (1976) A highly efficient method for washing mammalian spermatozoa. J. Reprod. Fert. 48, 347-353.

Harrison, R.A.P., Dott, H.M. \& Foster, G.C. (1978) Effect of ionic strength, serum albumin and other macromolecules on the maintenance of motility and the surface of mammalian spermatozoa in a simple medium. J. Reprod. Fert. 52, 65-73.

Mann, T. (1964) The Biochemistry of Semen and of the Male Reproductive Tract. Methuen, London.

Shannon, P. (1965a) Presence of a heat-labile toxic protein in bovine seminal plasma. J. Dairy Sci. 48, 13621365.

Shannon, P. (1965b) Contribution of seminal plasma, sperm numbers and gas phase to dilution effects of bovine spermatozoa. J. Dairy Sci. 48, 1357-1361.

Snedecor, G.W. \& Cochran, W.G. (1972) Statistical Methods. Iowa State University Press, Ames.

Received 24 August 1982 